\title{
A CLASS OF SETS WITH POSITIVE LENGTH AND ZERO ANALYTIC CAPACITY
}

\author{
PERTTI MATTILA
}

1. Introduction. A compact subset $E$ of the complex plane $C$ is said to be removable for bounded analytic functions if for any open set $U$ containing $E$ every bounded analytic function in $U \backslash E$ has an analytic extension to $U$. It is not hard to see that this condition is independent of $U$, see [Z, Appendix II]. Thus, by Liouville's theorem, $E$ is removable if and only if the only bounded analytic functions in $C \backslash E$ are the constant functions. The removability property can be formulated in terms of the analytic capacity $\gamma(E)$;

$$
\gamma(E)=\sup _{f} \lim _{z \rightarrow \infty}|z f(z)|
$$

where the supremum is taken over all functions $f$ which are analytic in $C \backslash E$ and for which $|f(z)| \leqq 1$ for $z \in C \backslash E$ and $\lim _{z \rightarrow \infty} f(z)=0$. Then $E$ is removable if and only if $\gamma(E)=0$, see [G2, p. 10] or [Z, p. 138].

A classical sufficient geometric condition, essentially due to Painlevé, for $\gamma(E)=0$ is $H^{1}(E)=0$ where $H^{1}$ is the one-dimensional Hausdorff (i.e. length) measure, [G2, p. 1]. This is not necessary; examples of sets $E$ with $\gamma(E)=0$ and $H^{1}(E)>0$ have been given by Vituškin [V1] and Garnett [G1]. In this paper we give geometric conditions which imply $\gamma(E)=0$ and apply to a large class of sets with $0<H^{1}(E)<\infty$. It can be checked that both Vituškin's and Garnett's examples satisfy these conditions. In fact, Garnett's example, the product of the 1/4-Cantor set with itself, is a particular case of a self-similar set with finite $H^{1}$ measure, which all are removable by the corollary in Section 4. However, in [G2, IV. 2] Garnett showed with a modified Cantor construction that there are removable sets with non- $\sigma$-finite $H^{1}$ measure. These are not covered by the results of this paper.

The sets considered in the main theorem in Section 3 are irregular in the sense of Besicovitch [B1], [B2], and thus have the property that their orthogonal projections on almost all lines through the origin are of measure zero. In [V2] Vituškin conjectured that this condition alone would be both necessary and sufficient for removability. Under the additional condition $H^{1}(E)<\infty$ this would mean that $E$ is removable if and only if $E$ is irregular. To one direction this is known: If $H^{1}(E)<\infty$ and $\gamma(E)=0$, then $E$ is irregular. In fact, if $E$ were not irregular, it would intersect some rectifiable curve in a set of positive $H^{1}$ measure. On the other hand, a 
compact subset $F$ of a rectifiable curve is removable if and only if $H^{1}(F)=0$, see [MD].

I want to thank B. Øksendal for bringing this field of problems to my attention and the referee for his useful comments.

2. Irregular sets. Let $E \subset C$ with $H^{1}(E)<\infty$. The upper and lower 1-dimensional densities of $E$ at a point $a \in C$ are

and

$$
\bar{D}(E, a)=\lim _{r \downarrow 0} \sup H^{1}(E \cap B(a, r)) /(2 r)
$$

$$
\underline{D}(E, a)=\liminf _{r \downarrow 0} H^{1}(E \cap B(a, r)) /(2 r),
$$

where $B(a, r)$ is the closed disc with centre $a$ and radius $r$. Besicovitch defined $E$ to be irregular if and only if $D(E, a)<1$ for $H^{1}$ almost all $a \in E$. One of his most important results is that this is equivalent with the property that the orthogonal projection of $E$ on almost every line through the origin is of measure zero, see [B2]. Besicovitch also characterized irregular sets as those which have approximate measure-theoretic tangent almost nowhere and which intersect every rectifiable curve in a set of measure zero [B1]. An exposition of Besicovitch's theory will appear in a forthcoming book by Falconer [FK].

For $0 \leqq \theta<2 \pi$ and $a \in C$ let $H_{\theta}(a)$ be the half-plane $\left\{z: \operatorname{Im} \mathrm{e}^{-i \theta}(z-a) \leqq 0\right\}$. We shall use the following two density theorems, see [FH, 2.10.19] and [B1, p. 317]:

(1) Suppose $E \subset C$ is $H^{1}$ measurable with $H^{1}(E)<\infty$. Then

$$
1 / 2 \leqq \bar{D}(E, a) \leqq 1 \text { for } H^{1} \text { almost all } a \in E .
$$

(2) If $E$ is irregular, then for every $\theta \in[0,2 \pi)$

$$
\underline{D}\left(E \cap H_{\theta}(a), a\right)=0 \text { for } H^{1} \text { almost all } a \in E .
$$

We say that $\theta \in[0, \pi)$ is a weak tangent direction for $E$ at $a$ if $\underline{D}(E, a)>0$ and for all $\delta>0$

$$
\liminf _{r \downarrow 0} r^{-1} H^{1}\left\{z \in E \cap B(a, r):\left|\operatorname{Im} e^{-i \theta}(z-a)\right| \geqq \delta|z-a|\right\}=0 .
$$

Note that by replacing lim inf by lim sup we would get Besicovitch's definition for the line through $a$ in the direction $\theta$ to be a tangent for $E$.

It is possible that a set has one, several or none weak tangent directions at its points. One can even construct sets $E$ such that $H^{1}(E)<\infty$, and for every $a \in E$, $D(E, a)>0$ and all directions $\theta \in[0, \pi)$ are weak tangent directions for $E$ at $a$. To such sets our theorem does not apply.

It follows immediately from the definition that given $a \in \boldsymbol{C}$ the set of those $\theta \in[0, \pi)$ which are not weak tangent directions for $E$ at $a$ is open in $[0, \pi)$. Thus if $A$ is an $H^{1}$ measurable subset of $E$ with $H^{1}(A)>0$ and if for $H^{1}$ almost all $a \in A$ there is such a direction, then the set of all pairs $(a, \theta) \in A \times[0, \pi)$ for which $\theta$ is not a weak tangent direction for $E$ at $a$ has positive $H^{1} \times H^{1}$ measure. 
3. The main result. We shall now prove the main theorem:

Theorem. Let $E$ be a compact irregular subset of $C$ with $H^{1}(E)<\infty$. Suppose thut for $H^{1}$ almost all $a \in E, D(E, a)>0$ and there is $\theta \in[0, \pi)$ which is not a weak tangent direction for $E$ at $a$. Then $\gamma(E)=0$.

Proof. Let $f$ be an analytic function in $C \backslash E$ with $|f(z)| \leqq 1$ and $\lim _{z \rightarrow \infty} f(z)=0$. It suffices to prove that $f$ vanishes identically. We first show that there is an $H^{\mathbf{1}}$ measurable function $\varphi: E \rightarrow C$ such that

$$
f(z)=\int_{E} \frac{\varphi(\zeta)}{\zeta-z} d H^{1} \zeta \quad \text { for } \quad z \in C \backslash E,
$$

and

$$
|\varphi(\zeta)| \leqq 1 \text { for } \zeta \in E .
$$

Since $H^{1}(E)<\infty$ we find with the help of [G2, II.3.1] a finite complex Borel measure $\mu$ with support on $E$ such that

$$
f(z)=\int \frac{d \mu \zeta}{\zeta-z} \text { for } \quad z \in C \backslash E .
$$

Using [G2, II.2.1] we see that $\mu(D)=-\int_{\partial D} f(\zeta) d \zeta /(2 \pi i)$, whence

$$
|\mu(D)| \leqq H^{1}(\partial D) /(2 \pi),
$$

whenever $D$ is a disc with $\int_{\partial D} \int|\zeta-z|^{-1} d|\mu| \zeta d H^{1} z<\infty$. Here $|\mu|$ denotes the total variation measure of $\mu$. Using Fubini's theorem one sees that this condition holds for almost all discs with fixed centre. From this it follows by approximation that (3) actually holds for all discs.

Using the definition of $H^{1}$ one derives from (3) that $|\mu|(A) \leqq H^{1}(A)$ for any Borel set $A \subset C$. Therefore $\mu$ is absolutely continuous with respect to $H^{1}$, and moreover the Radon-Nikodým derivative $\varphi$ satisfies $|\varphi| \leqq 1$, that is, (1) and (2) hold.

If $H^{1}(E)=0$ or $\varphi(z)=0$ for $H^{1}$ almost all $z \in E$, we are done. So we assume that $H^{1}(E)>0$ and that $\varphi$ does not vanish $H^{1}$ almost everywhere on $E$, and we complete the proof by showing that then $f$ cannot be bounded.

According to [FH, 2.8.18 and 2.9.13] $\varphi$ is approximately continuous at $H^{1}$ almost all $a \in E$ in the sense that for all $\varepsilon>0$

$$
\lim _{r \downarrow 0} r^{-1} H^{1}\{z \in E \cap B(a, r):|\varphi(z)-\varphi(a)| \geqq \varepsilon\}=0 .
$$

Using 2(1), 2(2) and the assumptions of the theorem, we find such a point $a$ and $\theta \in[0, \pi)$ with the additional properties that $\varphi(a) \neq 0$,

$$
\begin{gathered}
0<\underline{D}(E, a) \leqq \bar{D}(E, a) \leqq 1, \\
\underline{D}\left(E \cap H_{\theta}(a), a\right)=0,
\end{gathered}
$$

and that $\theta$ is not a weak tangent direction for $E$ at $a$. In fact, we are using the last remark of Section 2 with $A=\{a: \varphi(a) \neq 0\}$ to ensure that the set of all pairs $(a, \theta)$ 
with the above properties has positive $H^{1} \times H^{1}$ measure. Without loss of generality we may assume that $a=0, \varphi(0)=\beta>0$ and $\theta=0$.

Since 0 is not a weak tangent direction at the origin, there is $\delta, 0<\delta<1 / 2$, such that

where

$$
\alpha=\liminf _{r \downarrow 0} r^{-1} H^{1}(E \cap C(r))>0,
$$

$$
C(r)=\{z: \delta|z| \leqq|\operatorname{Im} z|,|z| \leqq r\} .
$$

Let $m$ be a positive integer. We also denote

$$
\begin{gathered}
B(r)=B(0, r), \\
C=\{z: \delta|z| \leqq \operatorname{Im} z\}, \\
D=\{z: 0 \leqq \operatorname{Im} z<\delta|z|\}, \\
H=H_{0}(0)=\{z: \operatorname{Im} z \leqq 0\}, \\
t=\alpha / 16, \\
\varepsilon=2^{-14} \delta \alpha \beta t^{m}, \\
u=\operatorname{Re} \varphi, \quad v=\operatorname{Im} \varphi, \\
F=\{z \in E: u(z) \geqq \beta / 2,|v(z)| \leqq \varepsilon\} .
\end{gathered}
$$

Using (4)-(7) we find $S>0$ such that for all $0<r \leqq S$

$$
\begin{gathered}
H^{1}((E \backslash F) \cap B(r)) \leqq \varepsilon r, \\
H^{1}(E \cap B(r)) \leqq 4 r, \\
H^{1}(E \cap H \cap B(S)) \leqq \varepsilon S, \\
H^{1}(E \cap C(r)) \geqq \alpha r / 2 .
\end{gathered}
$$

Denote $s=t^{m} S$ and for $j=1, \ldots, m$

Then

$$
R_{j}=E \cap B\left(t^{j-1} S\right) \backslash B\left(t^{j} S\right) .
$$

$$
\begin{gathered}
H^{1}\left(R_{j} \cap F \cap C\right) \\
\geqq H^{1}\left(E \cap C\left(t^{j-1} S\right)\right)-H^{1}\left(E \cap B\left(t^{j} S\right)\right)-H^{1}((E \backslash F) \cap B(S))-H^{1}(E \cap H \cap B(S)) \\
\geqq \alpha t^{j-1} S / 2-4 t^{j} S-2 \varepsilon S=\left(\alpha / 2-4 t-2 \varepsilon t^{1-j}\right) t^{j-1} S \geqq 2^{-3} \alpha t^{j-1} S .
\end{gathered}
$$

Let $z \in B(\delta s / 2) \backslash E$. If $|\zeta| \geqq s$ and $\operatorname{Im} \zeta \geqq \delta|\zeta|$, an elementary calculation gives

$$
-\operatorname{Im} \frac{1}{\zeta-z} \geqq \frac{\delta}{8|\zeta|} \text { and } \frac{1}{|\zeta-z|} \leqq \frac{2}{|\zeta|} \text {. }
$$


Using these inequalities and (9) we estimate

$$
\begin{gathered}
-\operatorname{Im} \int_{R_{j} \cap F \cap C} \frac{u(\zeta)}{\zeta-z} d H^{1} \zeta \geqq \frac{\delta \beta}{16} \int_{R_{j} \cap F \cap C} \frac{1}{|\zeta|} d H^{1} \zeta \\
\geqq 2^{-4} \delta \beta\left(t^{j-1} S\right)^{-1} 2^{-3} \alpha t^{j-1} S=2^{-7} \delta \alpha \beta, \\
\left|\int_{R_{j} \cap F \cap C} \frac{v(\zeta)}{\zeta-z} d H^{1} \zeta\right| \leqq 2 \varepsilon \int_{R_{j}} \frac{1}{|\zeta|} d H^{1} \zeta \\
\quad<2 \varepsilon\left(t^{j} S\right)^{-1} 4 t^{j-1} S=8 \varepsilon t^{-1} \leqq 2^{-8} \delta \alpha \beta,
\end{gathered}
$$

whence

$$
-\operatorname{Im} \int_{R_{j} \cap F \cap C} \frac{\varphi(\zeta)}{\zeta-z} d H^{1} \zeta \geqq 2^{-8} \delta \alpha \beta
$$

From (8) and (10) we get

so that

$$
\begin{aligned}
& H^{1}\left(R_{j} \backslash F\right) \leqq \varepsilon t^{j-1} S \leqq \varepsilon S, \\
& H^{1}\left(R_{j} \cap H\right) \leqq \varepsilon S,
\end{aligned}
$$

$$
\left|\int_{\left(R_{j} \backslash F\right) \cup\left(R_{j} \cap H\right)} \frac{\varphi(\zeta)}{\zeta-z} d H^{1} \zeta\right| \leqq 2\left(t^{j} S\right)^{-1} 2 \varepsilon S \leqq 2^{-10} \delta \alpha \beta .
$$

Let $\sigma=2^{-14} \delta \alpha \beta t$. By simple calculation $-\operatorname{Im} 1 /(\zeta-z) \geqq-\sigma /|\zeta|$ whenever $|z| \leqq$ $\sigma|\zeta| / 4$ and $\operatorname{Im} \zeta \geqq 0$. It follows that for all $\zeta \in F \cap D \backslash B(s)$ and $z \in B(\sigma s / 4) \backslash E$

Hence

$$
\begin{aligned}
& -\operatorname{Im} \frac{\varphi(\zeta)}{\zeta-z}=-u(\zeta) \operatorname{Im} \frac{1}{\zeta-z}-v(\zeta) \operatorname{Re} \frac{1}{\zeta-z} \\
& \geqq-\sigma|| \zeta|-2 \varepsilon /| \zeta\left|\geqq-2^{-12} \delta \alpha \beta t /\right| \zeta \mid .
\end{aligned}
$$

$$
-\operatorname{Im} \int_{R_{j} \cap F \cap D} \frac{\varphi(\zeta)}{\zeta-z} d H^{1} \zeta \geqq-2^{-12} \delta \alpha \beta t\left(t^{j} S\right)^{-1} 4 t^{j-1} S=-2^{-10} \delta \alpha \beta .
$$

Putting together the estimates (12)-(14) we obtain

Consequently,

$$
-\operatorname{Im} \int_{R_{j}} \frac{\varphi(\zeta)}{\zeta-z} d H^{1} \zeta \geqq 2^{-9} \delta \alpha \beta
$$

$$
-\operatorname{Im} \int_{E \cap B(S) \backslash B(s)} \frac{\varphi(\zeta)}{\zeta-z} d H^{1} \zeta \geqq m 2^{-9} \delta \alpha \beta
$$

Our final goal is to find $z$ so that $\left|\int_{E} \varphi(\zeta) /(\zeta-z) d H^{1} \zeta\right|$ is large. In view of (15) we still have to eliminate the possible cancellation coming from inside $B(s)$ and outside $B(S)$. For this we use the following simple lemmas: 
Lemma 1. Let $0<r<\infty, 0<\eta<1$ and $c>0$. If $\mu$ is a positive Borel measure on $C$ with $\mu(B(r)) \leqq c r$, then, $L^{2}$ denoting the Lebesgue measure on $C$,

$$
L^{2}\left\{z \in B(\eta r): \int_{B(r)} \frac{d \mu \zeta}{|\zeta-z|} \geqq M\right\} \leqq 2 \pi c \eta r^{2} / M .
$$

Proof. Let

$$
A=\left\{z \in B(\eta r): \int_{B(r)} \frac{d \mu \zeta}{|\zeta-z|} \geqq M\right\} .
$$

By Fubini's theorem,

$$
\begin{gathered}
M L^{2}(A) \leqq \int_{A} \int_{B(r)} \frac{d \mu \zeta}{|\zeta-z|} d L^{2} z=\int_{B(r)} \int_{A} \frac{d L^{2} z}{|\zeta-z|} d \mu \zeta \\
\leqq \int_{B(r)} \int_{B(\eta r)} \frac{d L^{2} z}{|z|} d \mu \zeta=2 \pi \eta r \mu(B(r)) \leqq 2 \pi c \eta r^{2} .
\end{gathered}
$$

Lemma 2. Let $g$ be an analytic function in $B(R)$. If $|g(z)| \leqq 2 N$ for $z \in B(R)$ and if there is $w \in B(R / 2)$ such that $|g(w)| \geqq N$, then

$$
L^{2}\{z \in B(R):|g(z)| \geqq N / 2\} \geqq \pi R^{2} / 16 .
$$

Proof. Let $A=\{z \in B(R):|g(z)| \geqq N / 2\}$. Using the mean value property we get for $0<r<R / 2$,

Hence

$$
\begin{gathered}
N \leqq|g(w)|=\left|\int_{\partial B(w, r)} g(\zeta) d H^{1} \zeta / 2 \pi r\right| \\
\leqq\left[(N / 2) H^{1}(\partial B(w, r) \backslash A)+2 N H^{1}(\partial B(w, r) \cap A)\right] / 2 \pi r \\
\leqq N / 2+(N / \pi r) H^{1}(\partial B(w, r) \cap A) .
\end{gathered}
$$

and

$$
H^{1}(\partial B(w, r) \cap A) \geqq \pi r / 2,
$$

$$
L^{2}(A) \geqq \int_{0}^{R / 2} H^{1}(\partial B(w, r) \cap A) d r \geqq(\pi / 2) \int_{0}^{R / 2} r d r=\pi R^{2} / 16 .
$$

We now complete the proof of the theorem. As $\bar{D}(E, 0) \leqq 1$ and $H^{1}(E)<\infty$ there is $c, 1 \leqq c<\infty$, independent of $m$, such that

$$
H^{1}(E \cap B(r)) \leqq c r \text { for all } 0<r<\infty .
$$

We choose $m$ so large that

$$
\begin{gathered}
M=m 2^{-10} \delta \alpha \beta>2 c / \eta \text { with } \eta=\sigma / 4, \\
N=M-4 c-1>16^{2} c .
\end{gathered}
$$

Since $M>2 c / \eta$ we can apply Lemma 1 to find $z_{0} \in B(\eta s) \backslash E$ such that

$$
\left|\int_{E \cap B(s)} \frac{\varphi(\zeta)}{\zeta-z_{0}} d H^{1 \zeta}\right| \leqq M .
$$


This combined with (15) gives

Write

$$
\left|\int_{E \cap B(S)} \frac{\varphi(\zeta)}{\zeta-z_{0}} d H^{1} \zeta\right| \geqq M .
$$

$$
f(z)=\int_{E \cap B(S)} \frac{\varphi(\zeta)}{\zeta-z} d H^{1} \zeta+\int_{E \cap B(2 S) \backslash B(S)} \frac{\varphi(\zeta)}{\zeta-z} d H^{1} \zeta+\int_{E \backslash B(2 S)} \frac{\varphi(\zeta)}{\zeta-z} d H^{1} \zeta,
$$

and observe that as $z_{0} \in B(S / 2)$

whence

$$
\left|\int_{E \cap B(2 S) \backslash B(S)} \frac{\varphi(\zeta)}{\zeta-z_{0}} d H^{1} \zeta\right| \leqq(2 / S) H^{1}(E \cap B(2 S)) \leqq 4 c,
$$

$$
\left|\int_{E \backslash B(2 S)} \frac{\varphi(\zeta)}{\zeta-z_{0}} d H^{1} \zeta\right| \geqq M-4 c-1=N .
$$

Next we show that there is $z_{1} \in B(S)$ such that

$$
\left|\int_{E \backslash B(2 S)} \frac{\varphi(\zeta)}{\zeta-z_{1}} d H^{1} \zeta\right|>2 N .
$$

If this is false, we can apply Lemma 2 to the function $g$,

to obtain

$$
g(z)=\int_{E \backslash B(2 S)} \frac{\varphi(\zeta)}{\zeta-z} d H^{1} \zeta,
$$

$$
L^{2}\{z \in B(S):|g(z)| \geqq N / 2\} \geqq \pi S^{2} / 16 .
$$

On the other hand, Lemma 1 implies

$$
\begin{gathered}
L^{2}\{z \in B(S) \backslash E:|f(z)-g(z)| \leqq N / 4\} \\
=L^{2}\left\{z \in B(S) \backslash E:\left|\int_{E \cap B(2 S)} \frac{\varphi(\zeta)}{\zeta-z} d H^{1} \zeta\right| \leqq N / 4\right\} \geqq \pi S^{2}(1-16 c / N) .
\end{gathered}
$$

From $N>16^{2} c$ we see that the sum of these two measures is greater than $\pi S^{2}$; therefore there is $z \in B(S) \backslash E$ such that $|g(z)| \geqq N / 2$ and $|f(z)-g(z)| \leqq N / 4$. But then $|f(z)| \geqq N / 4>1$, which is impossible. This proves (16).

Next we write

Here

$$
g(z)=\int_{E \cap B(4 S) \backslash B(2 S)} \frac{\varphi(\zeta)}{\zeta-z} d H^{1} \zeta+\int_{E \backslash B(4 S)} \frac{\varphi(\zeta)}{\zeta-z} d H^{1} \zeta
$$

whence

$$
\left|\int_{E \cap B(4 S) \backslash B(2 S)} \frac{\varphi(\zeta)}{\zeta-z_{1}} d H^{1} \zeta\right| \leqq(1 / S) H^{1}(E \cap B(4 S)) \leqq 4 c,
$$

$$
\left|\int_{E \backslash B(4 S)} \frac{\varphi(\zeta)}{\zeta-z_{1}} d H^{1} \zeta\right| \geqq 2 N-4 c \geqq N .
$$


Arguing as above we find $z_{2} \in B(2 S)$ such that

$$
\left|\int_{E \backslash B(4 S)} \frac{\varphi(\zeta)}{\zeta-z_{2}} d H^{1} \zeta\right|>2 N .
$$

Continuing in this manner we find for every $k$ a point $z_{k} \in B\left(2^{k-1} S\right)$ such that

$$
\left|\int_{E \backslash B\left(2^{k} S\right)} \frac{\varphi(\zeta)}{\zeta-z_{k}} d H^{1} \zeta\right|>2 N .
$$

But $E \backslash B\left(2^{k} S\right)=\emptyset$ for sufficiently large $k$, and we have arrived at a contradiciion.

4. Self-similar sets. Roughly, a set is self-similar if it can be decomposed into a finite number of parts geometrically similar to the whole set. Ordinary Cantor sets are examples. We consider only sets with Hausdorff dimension one, since those of dimension less than one are always removable by Painlevé's theorem, and those of dimension greater than one are never removable by [G2, III. 3.5]. The following definitions are due to Hutchinson $[\mathrm{H}]$.

Let $E$ be a compact subset of $C$ with Hausdorff dimension one. Suppose $E$ is self-similar, that is, there exist contracting similarity maps $S_{1}, \ldots, S_{n}: C \rightarrow C, n \geqq 2$, such that

$$
E=\bigcup_{i=1}^{n} S_{i} E \quad \text { and } \quad H^{1}\left(S_{i} E \cap S_{j} E\right)=0 \quad \text { for } \quad i \neq j .
$$

We also assume that the open set condition is satisfied: There is a bounded open non-empty set $O$ such that

$$
\bigcup_{i=1}^{n} S_{i} O \subset O \quad \text { and } \quad S_{i} O \cap S_{j} O=\emptyset \text { for } i \neq j .
$$

This always holds if the $S_{i} E$ 's are disjoint, cf. [H, 5.2(2)]. We have by [H, 5.3(1)], $0<H^{1}(E)<\infty$ and $-(E, a)>0$ for all $a \in E$.

By [MP, 4.3] there are only two possibilities: Either $E$ lies on a straight line, in which case it actually must be a line segment, or $E$ intersects every $C^{1}$ curve in a set of $H^{1}$ measure zero, which implies that it is irregular. In the second case it follows from [MP, 4.2] that $E$ has no weak tangent directions at all. Thus we have the following corollary to the theorem:

Corollary. Let $E$ be a compact subset of $\boldsymbol{C}$ with Hausdorff dimension one. Suppose that $E$ is not a line segment, $E$ is self-similar, and that the open set condition is satisfied. Then $\gamma(E)=0$. 


\section{References}

[B1] Besicovitch, A. S.: On the fundamental geometrical properties of linearly measurable plane sets of points (II). - Math. Ann. 115, 1938, 296-329.

[B2] Besicovitch, A. S.: On the fundamental geometrical properties of linearly measurable plane sets of points (III). - Math. Ann. 116, 1939, 349-357.

[FK] FALCONER, K. J.: Geometry of fractal sets. - To appear.

[FH] FEDERER, H.: Geometric measure theory. - Die Grundlehren der mathematischen Wissenschaften 153. Springer-Verlag, Berlin-Heidelberg-New York, 1969.

[G1] Garnett, J.: Positive length but zero analytic capacity. - Proc. Amer. Math. Soc. 24, 1970, 696-699.

[G2] GARNeTT, J.: Analytic capacity and measure. - Lecture Notes in Mathematics 297. SpringerVerlag, Berlin-Heidelberg-New York, 1972.

[H] Hutchinson, J. E.: Fractals and self-similarity. - Indiana Univ. Math. J. 30, 1981, 713-747.

[MD] Marshall, D. E.: Removable sets for bounded analytic functions. - Linear and complex analysis problem book, 199 research problems. Lecture Notes in Mathematics 1043. Springer-Verlag, Berlin-Heidelberg-New York, 1984, 485-490.

[MP] Mattila, P.: On the structure of self-similar fractals. - Ann. Acad. Sci. Fenn. Ser. A I Math. 7, 1982, 189-195.

[V1] VitušKın, A. G.: Example of a set of positive length but of zero analytic capacity. - Dokl. Akad. Nauk SSSR 127, 1959, 246-249 (Russian).

[V2] VITUŠKIN, A. G.: Analytic capacity of sets in problems of approximation theory. - Uspehi Mat. Nauk 22:6, 1967, 141-199 (Russian).

[Z] Zalcman, L.: Analytic capacity and rational approximation. - Lecture Notes in Mathematics 50. Springer-Verlag, Berlin-Heidelberg-New York, 1968.

University of Helsinki

Department of Mathematics

SF-00100 Helsinki

Finland

Received 6 February 1984 